\title{
The PROs and cons of liver resection in colorectal cancer
}

Approximately $40 \%$ of patients with colorectal cancer (CRC) develop liver metastases at some point during the course of the disease. A third of these patients can undergo hepatic resection, and half of these patients will achieve a 5-year overall survival but, what is the impact on quality of life of this kind of surgery? Jane Blazeby and colleagues have assessed the quality of life of 232 patients who underwent resection for CRC metastases by using generic and a disease specific patient-reported outcome measures (PROs); these data were obtained from the patients through questionnaires that assessed several aspects of health including physical, emotional and social functions. "The most significant findings were that liver resection for CRC has a relatively small and shortlived detrimental impact on most aspects of quality of life," explains Blazeby. Because liver resection confers a chance of long-term survival, understanding the impact of surgery on PROs is critical as it provides information that can be used by surgeons when discussing the outcomes of this treatment with patients. However, the study also showed that despite the early recovery, some problems (abdominal pain and sexual disfunction) persisted 12 months after surgery. Blazeby explains how these problems highlight "the importance of using generic and disease-specific PROs as such issues may have passed undetected by standard clinical follow up or using generic PROs alone."

But what happens when resection of liver metastases is not possible? Over the past decades, several techniques for local tumour destruction-such as radiofrequency ablation (RFA) - have been developed, although chemotherapy is still the standard of care for patients for non-resectable disease. Theo Ruers

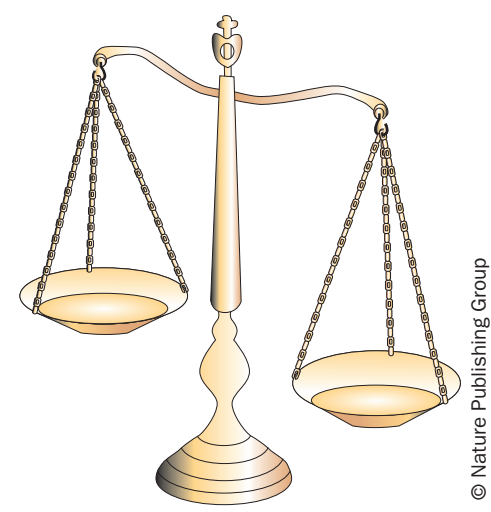

and colleagues have carried out a randomized phase II study in 119 patients to evaluate the efficacy of RFA plus chemotherapy (FOLFOX) versus chemotherapy alone. Selection criteria were no extrahepatic disease, less than 10 lesions, and lesions to be treated with RFA being smaller than $4 \mathrm{~cm}$."We observed that RFA plus chemotherapy resulted in a progression-free survival of 16.8 months versus 9.9 months in the control arm," says Ruers. Importantly, the study also showed that treatment with RFA was safe and patients recovered fast after the procedure. Although the study did not allow any comparison of overall survival in both arms "it gives sufficient reason to implement RFA now in clinical practice and treat patients with unresectable CRC liver metastases by RFA plus systemic treatment".

The findings from these two studies will help to understand the best ways to treat resectable and unresectable liver metastases in patients with CRC.

\section{Teresa Villanueva}

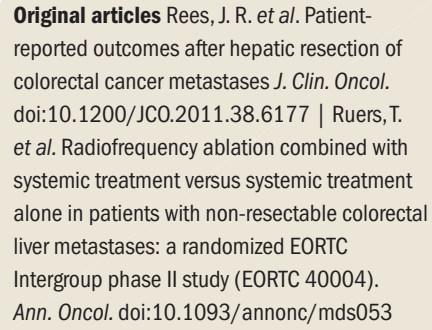

